\title{
Terapias complementares no enfrentamento do estresse no período puerperal: revisão integrativa da literatura
}

RESUMO | Objetivo: Identificar evidências científicas na literatura sobre o uso de terapias complementares no enfrentamento do estresse no período puerperal. Método : A coleta de dados foi realizada de janeiro a março de 2021. Trata-se de uma revisão integrativa da literatura, utilizou-se estratégia PICO para elaboração da pergunta de pesquisa: Quais são as evidências científicas encontradas na literatura sobre o uso de terapias complementares como benefício para enfretamento de estresse no período puerperal? Utilizou-se as bases de dados: SciELO; LILACS; MEDLINE; CINAHL e EMBASE. Os descritores utilizados segundo DESC/ MESH foram: Período Pós-Parto, Terapias Complementares, Estresse Emocional e Estresse Psicológico. Resultados: Encontrados 49 artigos, dos quais 6 selecionados, dentre as práticas utilizadas 6 terapias complementares distintas foram identificadas: biofeedback ou bioenergética; acupressão; reflexologia; musicoterapia e yoga. Conclusão: Os resultados dos estudos confirmaram a existência de evidências de que terapias complementares são benéficas para o enfrentamento do estresse no período puerperal. Descritores: Período Pós-Parto; Terapias Complementares; Estresse Emocional; Estresse Psicológico.

\begin{abstract}
To identify scientific evidence in the literature on the use of complementary therapies in coping with stress in the puerperal period. Method: Data collection was carried out from January to March 2021. This is an integrative literature review, using the PICO strategy to prepare the research question: What is the scientific evidence found in the literature on the use of therapies supplements as a benefit for coping with stress in the puerperal period? The following databases were used: SciELO; LILACS; MEDLINE; CINAHL and EMBASE. The descriptors used according to DESC/MESH were: Postpartum Period, Complementary Therapies, Emotional Stress and Psychological Stress. Results: 49 articles were found, 6 of which were selected, among the practices used, 6 distinct complementary therapies were identified: biofeedback or bioenergetics; acupressure; reflexology; music therapy and yoga. Conclusion: The results of the studies confirmed the existence of evidence that complementary therapies are beneficial for coping with stress in the puerperal period
\end{abstract}

Keywords: Postpartum period; Complementary Therapies; Emotional Stress; Psychological stress.

RESUMEN | Objetivo: Identificar evidencia científica en la literatura sobre el uso de terapias complementarias en el afrontamiento del estrés en el puerperio. Método: La recolección de datos se realizó de enero a marzo de 2021. Se trata de una revisión integradora de la literatura, utilizando la estrategia PICO para preparar la pregunta de investigación: ¿Cuál es la evidencia científica encontrada en la literatura sobre el uso de terapias complementarias como beneficio para el afrontamiento? con estrés en el puerperio? Se utilizaron las siguientes bases de datos: SciELO; LILACS; MEDLINE; CINAHL y EMBASE. Los descriptores utilizados según DESC / MESH fueron: Posparto, Terapias complementarias, Estrés emocional y Estrés psicológico. Resultados: se encontraron 49 artículos, de los cuales se seleccionaron 6, entre las prácticas utilizadas, se identificaron 6 terapias complementarias distintas: biofeedback o bioenergética; acupresión reflexología; musicoterapia y yoga. Conclusión: Los resultados de los estudios confirmaron la existencia de evidencia de que las terapias complementarias son beneficiosas para afrontar el estrés en el puerperio. Palabras claves: Período posparto; Terapias complementarias; Estrés emocional; Estrés psicológico.

\section{Izabel Dayana de Lemos Santos}

Enfermeira. Doutoranda em Enfermagem. Universidade Estadual de Londrina, Paraná, Brasil.

ORCID: 0000-0003-3559-5350

\section{Tatiane Tokushima}

Enfermeira Coordenadora do setor de obstetrícia do hospital São Francisco Instituto Vida. Mestre Universidade Estadual de Londrina, Paraná, Brasil.

ORCID: 0000-0002-8139-339X

\section{Franciane Maria da Silva Curan}

Enfermeira. Doutoranda em Enfermagem. Universidade Estadual de Londrina, Paraná, Brasil.

ORCID: 0000-0002-9786-5451

\section{Mariana Haddad Rodrigues}

Enfermeira. Pós- Doutoranda em Enfermagem. Universidade Estadual de Londrina, Paraná, Brasil.

ORCID: 0000-0002-8339-9760

\section{Fabiana Fontana Medeiros}

Enfermeira. Doutoranda em Enfermagem. Universidade Estadual de Londrina, Paraná, Brasil.

ORCID: 0000-0002-7876-572X

\section{Alexandrina Aparecida Maciel Cardelli}

Enfermeira. Doutora, Associada do Departamento de Enfermagem. Área da Saúde da Mulher e Gênero. Centro de Ciências da Saúde, Universidade Estadual de Londrina, Paraná, Brasil

ORCID:0000-0002-0222-8821 
INTRODUÇÃO

$\mathrm{D}$ urante a gestação, muitas modificações ocorrem no corpo da mulher, para poder acolher e nutrir o feto em desenvolvimento, alterações que acontecem de uma forma lenta e progressiva, cujo o desfecho é o parto, logo após o parto tem início o período puerperal que é uma fase do ciclo reprodutivo feminino repleta de modificações, diferente da gestação, são transformações que advém de forma abrupta, o organismo precisa se mobilizar para retornar o corpo pré-gravídico, no entanto além das modificações estruturais e hormonais, a mulher enfrenta transições de cunho emocionais e sociais. (1-3)

Além das transformações que ocorrem devido ao puerpério, a mulher precisa gerenciar adaptações da nova estrutura familiar e aos desafios em manter o bem estar geral do recém-nascido, que estão relacionados desde as necessidades fisiológicas básicas, assim como, os referentes ao processo de desenvolvimento e afeto do novo membro da família. São diversas circunstâncias que rodeiam o processo adaptativo do período puerperal, o que pode acarretar em situações repetidas de estresse. $(4,5)$

O estresse é um processo natural do organismo que ocorre em diversos momentos da vida, apesar de estar ligado à situação de perigo ou ameaça, encontra-se presente também em processos adaptativos intensos, como no período puerperal. O estresse está diretamente ligado a descargas de cortisol no organismo que em uma situação crônica, pode ter desfechos como hipertensão arterial, diabetes, além dos distúrbios psicológicos como ansiedade e depressão5-8.

Existem diversas maneiras de enfrentar as situações de estresse, prevenir danos à saúde tanto física como emocional e uma das formas que vem ganhando espaço no cotidiano da população em geral são as práticas de
Terapias Complementares (TC). As TC comtemplam o ser humano de uma forma integral e holística, visando um resultado positivo tanto na parte fisiológica como na psicológica, as técni-

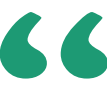

Além das transformações que ocorrem devido ao puerpério, a mulher precisa gerenciar adaptações da nova estrutura familiar e aos desafios em manter o bem estar geral do recémnascido, que estão relacionados desde as necessidades fisiológicas básicas, assim como, os referentes ao processo de desenvolvimento e afeto do novo membro da família

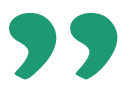

cas que incorporam as TC são milenares, no entanto foram incorporadas ao Sistema Único de Saúde (SUS) a pouco tempo. $(9,10)$

Em 2006 as TC foram incorporadas ao SUS, por meio da Política Nacional de Práticas Integrativas e Complemen- tares (PNPIC), que apresentou diretrizes norteadoras para medicina tradicional chinesa/acupuntura, homeopatia, plantas medicinais e fitoterapia, medicina antroposófica e termalismo social/crenoterapia. Em 2018, com objetivo de alcançar as metas determinadas pelo Organização Mundial de Saúde (OMS) em relação às $\mathrm{TC}$, foram incorporadas às políticas públicas mais técnicas de TC: apiterapia; aromaterapia; bioenergética; constelação familiar; cromoterapia; geoterapia; hipnoterapia; imposição de mãos; ozonioterapia e terapia de florais. (11-13)

Tem-se como pressuposto uma lacuna do conhecimento em relação à utilização de TC no período puerperal e em concordância com a literatura que um dos desfechos pode ser o estresse crônico que interfere diretamente em sua dinâmica de vida, acredita-se que as terapias complementares podem ser uma alternativa benéfica nessa fase do ciclo reprodutivo da mulher. Diante do exposto, surgiu a motivação para realizar uma revisão integrativa para compilar os estudos que investigam as práticas de TC que auxiliam no enfretamento do estresse no período puerperal, assim essa pesquisa tem como objetivo identificar evidências científicas na literatura sobre o uso de terapias complementares no enfrentamento do estresse no período puerperal.

\section{MÉTODO}

Trata-se de uma revisão integrativa da literatura, método que reúne, sintetiza e analisa os resultados de uma pesquisa minuciosa sobre um tema especifico, que contribui para o conhecimento aprofundado sobre a indagação levantada, organizado em seis fases: a primeira fase está relacionada à identificação do tema e seleção da hipótese assim como à elaboração da pergunta de pesquisa; a segunda fase estabelece critérios de inclusão e exclusão dos estudos pesquisados; terceira fase, é o 
momento da busca na literatura; quarta fase, etapa na qual serão extraído os dados e análise dos estudos dos estudos incluídos na revisão; quinta fase, diz respeito à interpretação e síntese dos resultados; sexta e última fase apresentação da revisão.( 14)

Como método para elaboração da pergunta de pesquisa foi utilizada a estratégia PICO, acrônimo para patient (paciente), intervention (intervenção), comparison (comparação), outcomes (resultados). O uso dessa estratégia contribui para identificação de descritores que facilitam a busca de estudos importantes nas bases de dados para composição da revisão integrativa. Dessa maneira a pergunta de pesquisa elaborada foi: Quais são as evidências científicas encontradas na literatura sobre o uso de terapias complementares como benefício para enfretamento de estresse no período puerperal? O primeiro item da estratégia $(P)$ se refere a puérperas; o segundo (I) a terapias complementares; o terceiro item da estratégia não foi utilizado nessa revisão integrativa para compor a pergunta de pesquisa; o quarto e último itens $(\mathrm{O})$ estão relacionados aos benefícios para o enfrentamento do estresse. (15)

A busca dos estudos foi realizada de janeiro a março de 2021, nas seguintes bases de dados e/ou bibliotecas eletrônicas: Scientific Eletronic Library Online (SciELO); Literatura Latino-Americana e do Caribe em Ciências da Saúde (LILACS); Medical Literature Analysis and Retrieval System Online (MEDLINE/PubMed); Cumulative Index to Nursing and Allied Health Literature (CINAHL) e Excerpta Medica data-base (EMBASE/ Emtree).

Os descritores controlados foram selecionados em consonância com o vocabulário dos Descritores em Ciências da Saúde (DECS) e Medical Subject Headings (MeSH), nos idiomas português, inglês, espanhol e francês, sendo elas: Período Pós-Parto (Postpartum Period, Período posparto, Pé- riode post-partum); Terapias Complementares (Complementary Therapies,
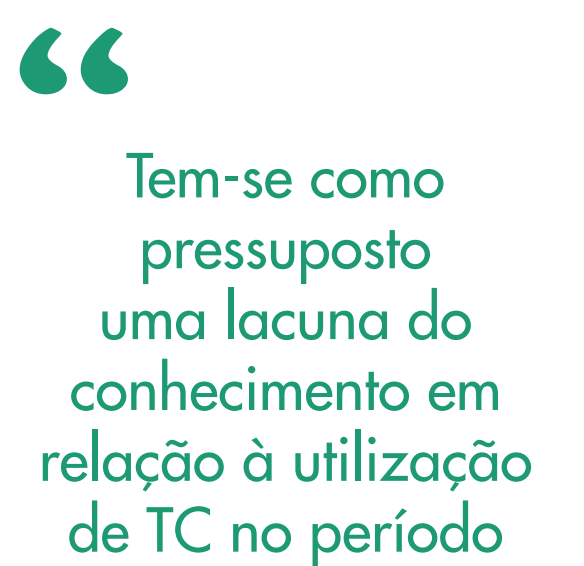 puerperal e em concordância com a literatura que um dos desfechos pode ser o estresse crônico que interfere diretamente em sua dinâmica de vida, acredita-se que as terapias complementares podem ser uma alternativa benéfica nessa fase do ciclo reprodutivo da mulher

Terapias complementarias, Thérapies complémentaires); Estresse Emocional (Emotional Stress, Estrés emocional,
Stress émotionnel); Estresse Psicológico (Psychological stress, Estrés psicológico, Stress psychologique). Os termos foram combinados de diferentes formas, para garantir uma busca ampliada do tema, entre as combinações foi utilizado o operador booleano AND, cujo o resultado do cruzamento foi utilizado em todas as bases de dados da seguinte forma: ((Período Pós-Parto) AND (Terapias Complementares) AND (Estresse Emocional)); ((Postpartum Period) AND (Complementary Therapies) AND (Stress, Psychological)); ((Periodo Posparto) AND (Terapias Complementarias) AND (Estrés Psicológico)); ((Période post-partum) AND (Thérapies complémentaires) AND (Stress psychologique)); ((Período Pós-Parto) AND (Terapias Complementares) AND (Estresse Psicológico)); ((Postpartum Period) AND (Complementary Therapies) AND (Emotional Stress)); ((Periodo Posparto) AND (Terapias Complementarias) AND (Estrés emocional)); ((Période post-partum) AND (Thérapies complémentaires) AND (Stress émotionnel)), esse padrão de busca foi seguido em todas as bases de dados.

Os critérios de inclusão definidos para análise dos estudos foram: grupo populacional de mulheres no período puerperal; publicados entre os anos de 2000 a 2020; disponíveis na integra; que apresentassem o uso de terapias complementares no enfrentamento do estresse puerperal. Foram excluídas revisões tradicionais de literatura, estudos secundários, carta-resposta, editoriais, teses, dissertações, artigos de opinião, artigos duplicados e que não respondessem à pergunta de pesquisa. Não houve limitação de idiomas devido à escassa produção científica sobre o tema.

Foram identificados um total de 49 artigos, desses um foi excluído por duplicidade, resultando em 48 artigos únicos, posteriormente foi realizada a leitura dos títulos e resumos obedecendo os critérios determinados para 


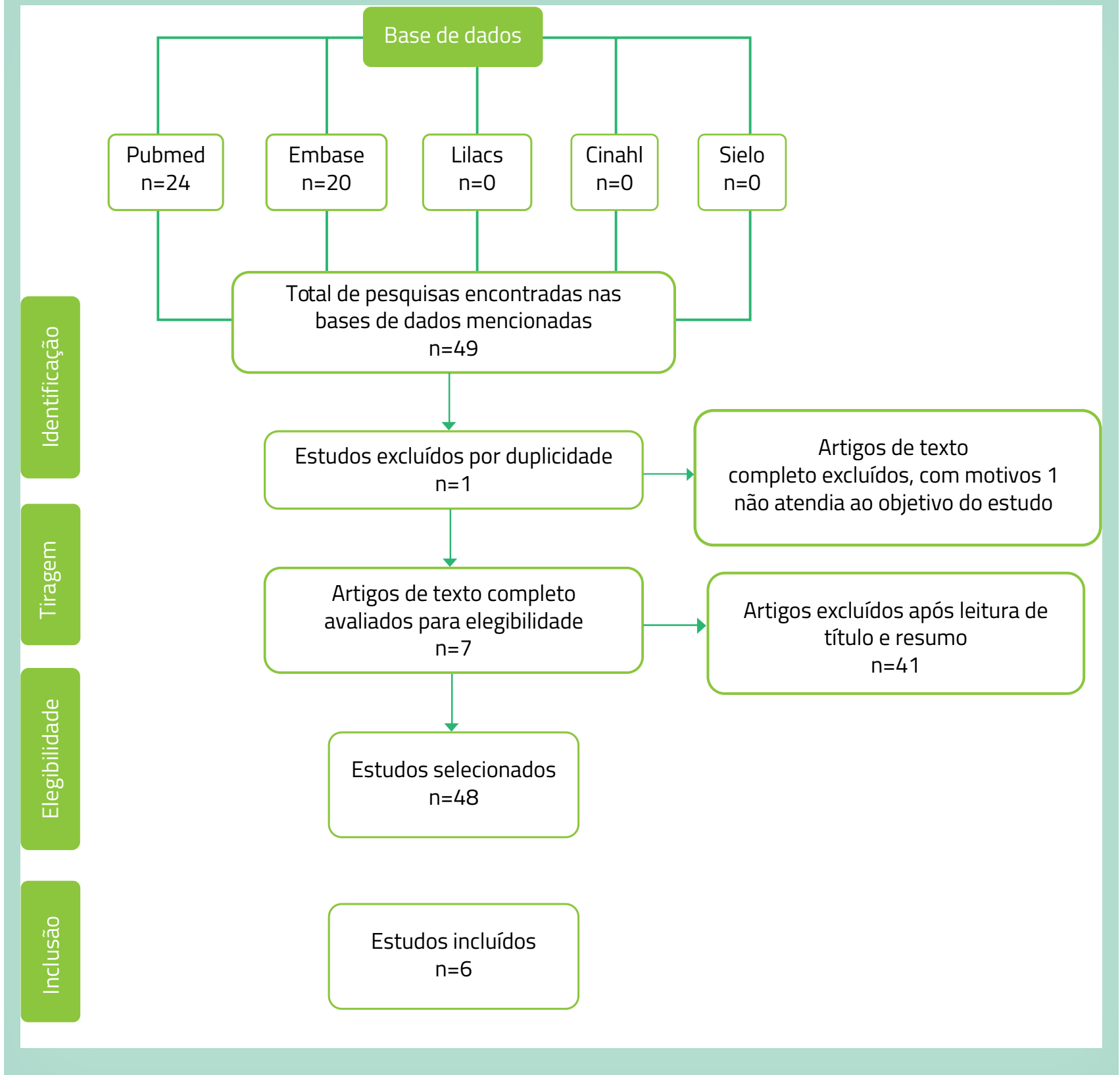

Fonte: Elaborada pelos autores, 2021

inclusão e exclusão dos estudos, após a finalização dessa etapa 41 artigos foram excluídos, 7 artigos se adequaram aos critérios de elegibilidade, no entanto após a leitura na integra das pesquisas 2 artigos foram excluídos, um por usar uma pratica não reconhecida no Brasil como terapia complementar e outra por que não respondia de forma especifica a pergunta norteadora da revisão integrativa, o que resultou em uma amostra total de 6 artigos (Figura 1).

Para coleta de dados, utilizou-se um instrumento estruturado desenvolvido pelo pesquisador principal, formado por itens relacionados à identificação do artigo, sendo estes o título, os autores, o ano de publicação e país em que a pesquisa foi realizada, assim como, o tipo de estudo e Nível de Evidência (NE), objetivo, método e materiais, desfecho principal.

Para determinar o NE foi utilizado 
um sistema de hierarquização, segundo conceitos empregados por estudiosos da área da enfermagem, baseado na força da evidencia científica, categorizado em sete níveis: nível 1 (mais fraco), evidências provenientes de revisão sistemática ou metanálise de ensaios clínicos randomizados controlados ou de diretrizes clínicas baseadas em revisões sistemáticas de ensaios clínicos randomizados controlados; nível 2, evidências derivadas de pelo menos um ensaio clínico randomizado controlado e bem delineado; nível 3, evidências obtidas em ensaios clínicos bem de- lineados, sem randomização; nível 4, evidências provenientes de estudos de coorte e de caso-controle bem delineados; nível 5, evidências derivadas de revisão sistemática de estudos descritivos e qualitativos; nível 6, evidências derivadas de um único estudo descritivo ou qualitativo; nível 7, evidências oriundas de opinião de autoridades ou relatório de especialistas. $(16,17)$

\section{RESULTADOS}

Amostra foi composta por 6 artigos científicos que respondiam especifica- mente ao objetivo da revisão integrativa, para melhor análise dos dados foi construído um quadro que permitiu reunir e epilogar as informações, tais como: identificação do artigo (autoria, ano de publicação, país no qual foi realizada a pesquisa, idioma de vinculação e título do estudo); e características metodológicas (tipo do estudo, nível de evidencia cientifica, objetivo, método e desfecho principal). Apresentados no Quadro 1 conforme o ano de publicação.

Os estudos incluídos na amostra foram publicados no ano de $2010(n=1)$,

Quadro 1: Síntese dos estudos primários incluídos na revisão integrativa (n=6), Londrina, PR, 2021.

$\begin{array}{ccccc}\text { Autor / } & \text { Título do } & \text { Tipo De } & & \\ \text { Ano /País / } & \text { artigo } & \text { estudo/ } & \text { Objetivo } & \text { Método }\end{array}$

Tseng, Y F; Efeitos de ouvir Chen, $\mathrm{CH}$; música nos níLee, CCS veis de estresse 2010 Chi- e ansiedade no na Inglês
Ensaio clínico randomizado $(n=77)$

NE 2
Investigou o efeito de ouvir música relaxante especialmente selecionada em casa, de forma autorregulada, teve sobre 0 estresse percebido e o estado de ansiedade no período puerperal
Grupo experimental ouviu música em casa por pelo menos 30 minutos por dia durante duas semanas e recebeu cuidados pós-parto. Associado a aplicação da Escala de Estresse Percebido
Este estudo não forneceu evidências de que a música de designer pré-selecionada reduziu os níveis de estresse e ansiedade entre Mulheres no período puerperal
Shin, HS; Efeitos da tera- Ensaio

Ryu, KH; pia do riso na clínico não

Song, Y A fadiga pós-parto

2011

Coreia

Coreano randomi-

zado

$(\mathrm{n}=67)$

NE 3 e respostas ao estresse de Mulheres no pós-parto
Examinar os efeitos da terapia do riso na fadiga e no estresse em mulheres no pós parto

\section{0 grupo experimental}

recebeu terapia do riso de um especialista por 60 minutos, duas vezes por semana durante 2 semanas, um total de 4 sessões, associado questionário e coleta de cortisol do leite materno
Terapia do riso tem um efeito positivo na redução da fadiga e do estresse em mulheres no pós-parto
Intervenção de

Kudo, N; Biofeedback na

Shinohara, Variabilidade do

H; Kodama, batimento cardíH aco para Redu2014 ção de estresse Japão psicológico duInglês rante o período pós-parto inicial
Examinar a

Caso controle $(n=55)$

NE 4 eficácia da intervenção de biofeedback na Variabilidade do batimento cardíaco para redução do estresse psicológico em mulheres no período pós-parto inicial
Implementação de

Biofeedback na Variabilidade do batimento cardíaco usando um dispositivo portátil e controle respiratório, no pedia pós parto. ríodo de um mês, a partir do quarto
As mulheres no pós-parto que implementaram o biofeedback para Variabilidade do batimento cardíaco após o parto, ficaram relativamente livres de ansiedade e reclamaram menos de dificuldades no sono um mês após o parto. 


\begin{tabular}{clc} 
Choi, M S; & Efeitos da mas- & \\
Lee, E J; & sagem de refle- & Caso \\
2015 & xologia podal na & controle \\
Coreia & fadiga, estresse & (n=70) \\
Coreano & e depressão em & NE 4 \\
& mulheres no & \\
& \multicolumn{2}{c}{ pós-parto }
\end{tabular}

Jung, G S;
In-Ryoung
Choi, I R;
Kang; H Y;
Choi, E Y
2017
Coreia
Inglês

Efeitos da massagem com acupressão nos meridianos na composição corporal, edema, em mulheres no pós-parto estresse e fadiga
Quase experimental $(n=39)$

NE 3
Identificar os efeitos da massagem de reflexologia podal na fadiga, estresse e depressão em mulheres no pós-parto
Massagem de reflexologia podal foi fornecida ao grupo experimental uma vez por dia durante três dias
0 nível de fadiga, nível de cortisol na urina e nível de depressão foi significativamente menor no grupo experimental menor do que no grupo controle
Investigar os efeitos da massagem de acupressão nos meridianos na composição corporal, edema, estresse e fadiga em mulheres no pós-parto.
0 grupo experimental recebeu massagem de acupressão nos meridianos por 90 minutos diários, por 5 dias.
0 grupo experimental demonstrou uma diminuição significativamente maior em comparação com o grupo de controle em medidas de composição corporal, edema, estresse subjetivo total, estresse psicológico e fadiga.

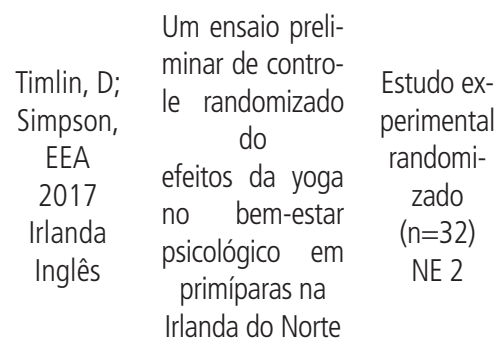

Fonte: Registro Hospitalar do Câncer, 2018.

$2011(n=1), 2014(n=1), 2015(n=1)$ e $2017(n=2)$, realizados em 4 países: Japão $(n=1)$, Coreia $(n=3)$, China $(n=1)$ e Irlanda $(n=1)$, dos quais 4 foram escritos e divulgados no idioma inglês e 2 em coreano. Quanto ao desenho de pesquisa 2 estudos utilizaram ensaio clinico randomizado (NE 2), 2 empregaram caso-controle (NE 4), 1 aplicou o método quase experimental (NE 3) e 1 utilizou ensaio clinico não randomizado (NE 3), todos métodos com aspectos quantitativos.

Dentre a terapias complementares utilizadas nas pesquisas, identificou-se 6 diferentes procedimentos: a atividade de biofeedback ou bioenergética; a técnica de acupressão, assim com a reflexologia; a musicoterapia; a prática de yoga e terapia por indução de risos. Dentre os desfechos principais a maior parte dos estudos apresentaram resultados positivos quando analisado a técnica utilizada, somente o estudo sobre os efeitos de ouvir música, não forneceram evidencias significativas.

\section{DISCUSSÃO}

Após a análise dos estudos incluídos na revisão integrativa, nota-se que a realização de pesquisas que respondem à questão norteadora, relacionadas à TC, estão concentradas em países asiáticos, pois foi também nessas regiões que a medicina tradicional chinesa (MTC) se iniciou. Foi a partir da MTC que diversas TC tiveram origem, são práticas milenares nos países asiáticos, no entanto apenas no final da década de 70 que a OMS elaborou um programa de Medicina Tradicional, com objetivo de incentivar e promover políticas e práticas nesta área. (18-23)

Mesmo com escassez de evidencias cientificas que respondam à pergunta da revisão integrativa, os estudo que foram incluídos tiveram um NE satisfatório, assim como um rigor metodológico aceitável, mesmo algumas pesquisas apresentando uma deficiência no cálculo amostral, percebe-se uma preocupação com os métodos para comprovação dos resultados. Dos seis artigos incluídos dois utilizaram como desenho de pesquisa o ensaio clinico randomizado, considerado método de condução de pesquisa padrão ouro para atestar o efeito de uma determinada terapêutica. (19, 24-25)

Entre as inúmeras TC reconhecidas pela OMS, seis práticas foram encontradas nessa revisão com objetivo de enfrentamento do estresse no período puerperal, a maioria das estratégias apresentaram resultados positivos, apenas um artigo não trouxe diferenças estatísticas significantes, foi o estudo direcionado à musicoterapia, todavia, 
os autores ressaltam a importância do olhar cuidadoso dos profissionais de saúde para as mulheres no enfrentamento do estresse no período puerperal. $(12,19)$

Dentre as outras TC encontradas nesta pesquisa todas tiveram resultados estatísticos significativamente positivos. Uma das técnicas mais interessantes e inusitadas a ter resultados asseverativos, foi a terapia do riso. Em relação ao sistema nervoso central (SNC) o riso pode trazer uma sensação de alegria, o que tem como resultado a liberação de dopamina, neurotransmissor responsável pela sensação de bem-estar, além da liberação da dopamina o riso induz liberação de serotonina e endorfinas dessa forma diminuindo os níveis de cortisol no organismo. $(6,20,26)$

A MTC, tem suas bases na concepção do ser humano como um ser indivisível, cujo o equilíbrio de suas energias reflete diretamente no bem-estar físico e emocional, assim visando um cuidado integral e holístico. Entre as demais TC encontradas, dois artigos se referem a práticas antigas e reconhecidas, como a acupressão, uma técnica de massagem parecida com a acupuntura, porém ao invés de utilizar agulhas no meridianos, que são os canais que ligam a superfície com órgão específicos, se utiliza a pressão com a ponta dos dedos, assim como a reflexologia que com pressões em determinados locais dos pés, os resultados positivos são observados em outros locais do corpo, são técnicas que promovem o relaxamento e bem estar, sensações diretamente ligadas à diminuição dos níveis de estresses 12, 22-23.

Outra prática com resultados confirmativos no enfrentamento do estresse no período puerperal foi a yoga, um exercício considerado psicofísico que traz equilíbrio e saúde para as dimensões físicas, mentais, emocionais e espirituais do indivíduo, está associado também à regulação do eixo hipotálamo-hipófise-adrenal (HPA) que corres- ponde à liberação de cortisol diante de um estressor. (27-29)

O estresse além de agir no HPA,

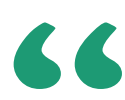

Nota-se uma diferença significativa entre os artigos identificados na base de dados e os incluídos no estudo, o que evidência uma dificuldade em elaborar pesquisa de revisão integrativa que elucidem questões pouco frequentes na literatura

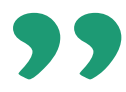

pode ter influencias significativas no sistema nervoso autonomo (SNA), mais especificamente no sistema nervoso simpatico (SNS) que é automaticamente acionado mediante situações de estresse, preparando o corpo para luta ou fuga aumentando a oferta de glicose e irrigação sanguinea para o organismo, processo pelo qual em situaçoes cronicas pode gerar consquencias indesejadas para o ser humano, uma estretegia que se mostrou efetiva para o equilibrio do SNS foi o Biofeedback, consiste no controle da respiração para modificar a variabilidade do batimentos cardiacos, sinalizando para o SNA que o SNS não precisa sera acionado, consequantemente retornando ao equilibrio das sisnalizações centrais para o organismo. $(21,30,31)$

\section{CONCLUSÕES}

As TC apesar de serem oriundas de conhecimentos milenares como a MTC, são pouco utilizadas no âmbito da saúde para estabilização, promoção e prevenção das diversas condições de desequilíbrio fisiológico, de cunho físico e emocional do ser humano. No entanto os resultados dos estudos primários incluídos na revisão integrativa, confirmam a existência de evidencias de que TC são benéficas para o enfrentamento do estresse no período puerperal.

Nota-se uma diferença significativa entre os artigos identificados na base de dados e os incluídos no estudo, o que evidência uma dificuldade em elaborar pesquisa de revisão integrativa que elucidem questões pouco frequentes na literatura.

Observando a escassez de pesquisas sobre o tema, espera-se que os resultados dessa pesquisa colaborem para que mais pesquisas utilizando as TC no enfrentamento do estresse puerperal sejam feitas e que possam ser ferramentas utilizadas pelos profissionais da saúde afim de propor uma experiência positiva do puerpério, para diversas mulheres. 


\section{Referências}

1. Elias EA, Pinho JP, Oliveira SR. Expectativas e sentimentos de gestantes sobre o puerpério: contribuições para a enfermagem. Enferm Foco [Internet]. 2021[cited 2021 Jan 14];12(2):283-9. DOI 10.21675/2357707X.2021.v12.n2.4058. Available from: http://revista.cofen.gov.br/index.php/enfermagem/article/view/4058/1132

2. Alves TV, Bezerra MMM. Principais alterações fisiológicas e psicológicas durante o Período Gestacional. Id on Line Rev. Mult. Psic. [Internet]. 2020 [cited 2021 Jan 10];14(49):114-126. DOI: https://doi.org/10.14295/ idonline.v14i49.2324. Available from: https://idonline.emnuvens.com.br/ id/article/view/2324/3608

3. Silva LP, Silveira LM, Mendes TJM, et al. Assistência puerperal e a construção de um fluxograma para consulta de enfermagem. Rev. Bras. Saúde Mater. Infant. [Internet]. 2020 [cited 2021 Jan 10];20(1):115-127. DOI: https://doi.org/10.1590/1806-93042020000100007. Available from: https:// www.scielo.br/j/rbsmi/a/jjsBnwhpS4K5FT4WMn8zH7d/abstract/?lang=pt

4. Silva DD, Lopes PES, Silva VR, et al. Principais dificuldades vivenciadas por primíparas no cuidado ao recém-nascido. Revista Eletrônica Acervo Saúde. [Internet]. 2021 [cited 2021 Jan 10];13(2). DOI: https://doi. org/10.25248/reas.e5489.2021 Available from: https://acervomais.com. br/index.php/saude/article/view/5489

5. Cunha ACB, Eroles NMS, Resende LM. "Tornar-se mãe": Alto nível de estresse na gravidez e maternidade após o nascimento. Interação em psicologia. [Internet]. 2020[cited 2021 Jan 10]; 24(03): 279-287. DOI: http:// dx.doi.org/10.5380/riep.v24i3.62768 Available from: https://revistas.ufpr. br/psicologia/article/view/62768

6. Nogueira ML. Rir é o melhor remédio: terapia do riso na atenção a pacientes em unidade de terapia intensiva. Temas em saúde. [Internet]. 2020[cited 2020 Nov 20]; 20(5):119-147. DOI: 10.29327/213319.20.57 Available from: https://temasemsaude.com/wp-content/uploads/2020/11/20507.pdf

7. Penteado MS, Oliveira TC. Associação estresse-diabetes mellitus tipo II. Rev Bras Clin Med. [Internet]. 2009 [cited 2020 Nov 20];7:40-45. Available from: http://files.bvs.br/upload/S/1679-1010/2009/v7n1/a40-45.pdf

8. Silva RM, Goulart CT, Guido LA. Evolução histórica do conceito de estresse. Rev. Cient Sena Aires. [Internet]. 2018 [cited 2020 Nov 20];7(2)14856. Available from: http://revistafacesa.senaaires.com.br/index.php/revisa/article/viewFile/316/225

9. Oliveira HF, Marques NA, Costa SR. et al. Integrative and complementary practices in health professionals: an experience report. Research, Societyand Development. [Internet]. 2020 [cited 2020 Nov 20]; 9(10). DOI: https://doi.org/10.33448/rsd-v9i10.8489. Available from: https://rsdjournal.org/index.php/rsd/article/view/8489

10. Dalmolin IS, Heidemann ITSB, Freita VL. Práticas integrativas e complementares no sistema único de saúde: desvelando poderes e limites. Rev. esc. doente USP. [Internet]. 2019 [cited 2020 Nov 20]; 53. DOI: https://doi. org/10.1590/S1980-220X2018026603506 Available from: https://www. scielo.br/j/reeusp/a/4KL44rcCykZzxdPPDZmfQZg/?lang=pt

11. Brasil. Ministério da Saúde. Portaria no 971, de 03 de maio de 2006. Aprova a Política Nacional de Práticas Integrativas e Complementares (PNPIC) no Sistema Único de Saúde. Diário Oficial da União, Brasília, DF, 03 mai. 2006.

12. WHO. World Health Organization. Who global report on traditional and complementary medicine 2019: 228.

13. Brasil. Ministério da Saúde. Secretaria de Atenção à Saúde. Depar- tamento de Atenção Básica. Política nacional de práticas integrativas e complementares no SUS: atitude de ampliação de acesso / Ministério da Saúde. Secretaria de Atenção à Saúde. Departamento de Atenção Básica. - 2. ed. - Brasília: Ministério da Saúde, 2015. 96 p

14. Mendes KDS, Silveira RCCP, Galvao CM. Revisão integrativa: método de pesquisa para a incorporação de evidências na saúde e na enfermagem. Texto contexto - enferm. [Internet].2008 [cited 2020 Nov 20];17(4) 758-764. DOI: https://doi.org/10.1590/S0104-07072008000400018 Available from: https://www.scielo.br/j/tce/a/XzFkq6tjWs4wHNqNjKJLkXQ/?lang=pt

15. Santos CMC, Pimenta CAM, Nobre MRC. A estratégia PICO para a construção da pergunta de pesquisa e busca de evidências. Rev. Latino-Am. Enfermagem. [Internet].2007[cited 2020 Nov 20];15(3):508-511. DOI: https://doi.org/10.1590/S0104-11692007000300023 Available from: https://www.scielo.br/j/rlae/a/CfKNnz8mvSqVjZ37Z77pFsy/?lan$\mathrm{g}=\mathrm{pt}$

16. Melnyk BM, Fineout-overholt E. Evidencebased practice in nursing \& healthcare. A guide to best practice. Philadelphia: Lippincot Williams \& Wilkins; 2005:3-24.

17. Galvão CM. Níveis de Evidência. Acta Paul Enferm. [Internet]. 2006 [cited 2021 Jan 20];19(2): Editorial. DOI: https://doi.org/10.1590/S010321002006000200001 Available from: https://www.scielo.br/j/ape/a/JXr$\mathrm{fXqCfD4vPztQFQBrkB7g/?lang=pt}$

18. Brasil. Ministério da Saúde. Portaria ${ }^{\circ}$ 702, de 21 de março de 2018. Altera a Portaria de Consolidação $n^{\circ} 2 / G M / M S$, de 28 de setembro de 2017, para incluir novas práticas na Política Nacional de Práticas Integrativas e Complementares - PNPIC. Diário Oficial da União, Brasília, DF, 21 mar. 2018.

19. Ying-fen T, Chung-hey C, Chihchen SL. Effects of listening to music on postpartum stress and anxiety levels. Journal of Clinical Nursing. [Internet]. 2010[cited 2021 Jan 20];19:1049-1055. DOI: 10.1111 / j. 1365-2702.2009.02998.x. Available from: https://onlinelibrary.wiley.com/ doi/10.1111/j.1365-2702.2009.02998.x

20. Shin HS, Ryu KH, Song YA. Effects of Laughter Therapy on Postpartum Fatigue and Stress Responses of Postpartum Women. J Korean Acad Nurs. [Internet]. 2011[cited 2021 Jan 20];41(3):294-301. DOI: 10.4040 I jkan.2011.41.3.294. Available from: https://www.jkan.or.kr/DOlx. php?id=10.4040/jkan.2011.41.3.294

21. Kudo N, Shinohara H, Kodama H. Heart Rate Variability Biofeedback Intervention for Reduction of Psychological Stress During the Early Postpartum Period. Appl Psychophysiol Biofeedback. [Internet]. 2014 [cited 2021 Jan 20];39: 203-211. DOI: 10.1007 / s10484-014-9259-4. Available from: https://www.ncbi.nlm.nih.gov/pmc/articles/PMC4220117/

22. Choi MS, Lee EJ. Effects of Foot-Reflexology Massage on Fatigue, Stress and Postpartum Depression in Postpartum Women. J Korean Acad Nurs. [Internet]. 2015[cited 2021 Jan 20];45(4) 587-594. DOI: 10.4040 I jkan.2015.45.4.587. Available from: https://www.jkan.or.kr/DOlx. php?id=10.4040/jkan.2015.45.4.587

23. Jung GS, Choi IR, Kang HY, Choi EY. Effects of Meridian Acupressure Massage on Body Composition, Edema, Stress, and Fatigue in Postpartum Women. The journal of alternative and complementary medicine. [Internet]. 2017 [cited 2021 Jan 20];00(00):1-7. DOI: https://doi. org/10.1089/acm.2016.0362. Available from: https://www.liebertpub. com/doi/10.1089/acm.2016.0362 
24. Timlin D, Simpson EEA. A preliminary randomised control trial of the effects of Dru yoga on psychological well-being in Northern Irish first time mothers. Midwifery. [Internet]. 2017[cited 2021 Jan 20];46:29-36. DOI: 10.1016 / j.midw.2017.01.005. Available from: https://www.sciencedirect.com/science/article/abs/pii/S0266613817300165?via\%3Dihub

25. Carvalho APV, Silva V, Grande AJ. Avaliação do risco de viés de ensaios clínicos randomizados pela ferramenta da colaboração Cochrane. Diagn Tratamento. [Internet] 2013[cited 2020 Nov 20];18(1):38-44. Available from: http://files.bvs.br/upload/S/1413-9979/2013/v18n1/a3444.pdf

26. Esperidião VA, Colombo MM, Monteverde DT. et al. Neurobiologia das emoções. Revista de Psiquiatria Clínica. [Internet]. 2008[cited 2020 Nov 20]; 35(2):55-65. DOI: https://doi.org/10.1590/S010160832008000200003. Available from: https://www.scielo.br/j/rpc/a/ t55bGGSRTmSVTgrbWvqnPTk/abstract/?lang=pt\&format=html

27. Ross $A$, Thomas $S$. The health benefits of yoga and exercise: A review of comparison studies. Journal of Alternative and Complementary Medicine. [Internet]. 2010 [cited 2020 Nov 20];16(1):3-12. DOI: https://doi. org/10.1089/acm.2009.0044. Available from: https://www.liebertpub. com/doi/abs/10.1089/acm.2009.0044

28. Araújo MAN, Prado BGL. A Prática do Yoga no Pré-natal: redução do estresse e outros achados. Revista Psicologia, Diversidade e Saúde. [Internet]. 2020[cited 2020 Nov 20]; 9(3):374-387. DOI: https://doi.org/10.17267/2317-3394rpds.v9i3.3051. Available from: https://www5. bahiana.edu.br/index.php/psicologia/article/view/3051

29. Aguiar CCV, Alves VH, Pereira AV, et al. Yoga como prática integrativa e recurso terapêutico no apoio ao aleitamento materno. Enferm Foco. [Internet]. 2021[cited 2020 Nov 20];12(2):237-43. DOI: 10.21675/2357707X.2021.v12.n2.3786. Available from: http://revista.cofen.gov.br/index.php/enfermagem/article/view/3786

30. Araújo LM, Carvalho CMS, Amaral MM, Santos L. Variabilidade da Frequência Cardíaca como biomarcador do estresse: revisão integrativa. Research, Society and Development. [Internet]. 2020[cited 2020 Nov 20]; 9(12). DOI: https://doi.org/10.33448/rsd-v9i12.11125. Available from: https://rsdjournal.org/index.php/rsd/article/view/11125

31. Silva EM, Reis DA. Fatores de risco cardiovascular em estudantes de enfermagem de uma universidade do interior do Amazonas. Revista Nursing. [Internet]. 2021[cited 2021 Dez 8]; 24 (280). DOI: https://doi. org/10.36489/nursing.2021v24i280p6221-6234. Available from: http:// revistas.mpmcomunicacao.com.br/index.php/revistanursing/article/ view/1779 